\title{
Recent advances in prophylactic human papillomavirus (HPV) vaccination: a review of key literature published between September 2017 and September 2018
}

\author{
Anja Šterbenc ${ }^{1}$, Polona J. Maver ${ }^{1}$, Mario Poljak ${ }^{1 凶}$
}

\begin{abstract}
Prophylactic human papillomavirus (HPV) vaccines represent a revolutionary approach in preventing and potentially eliminating HPV-related cancers. Overwhelming real-life data are confirming the high efficacy and exceptional safety profile of all three prophylactic HPV vaccines currently available, which was previously shown in pivotal clinical trials. In this review, we summarized and discussed in our opinion the most influential peer-reviewed literature published between September 2017 and September 2018; that is, during the $2017 / 2018$ school year in Slovenia. We mainly focus on publications on progress and the development of novel prophylactic HPV vaccines, efficacy clinical trials evaluating various dosing schemes and HPV vaccination of alternative populations, and studies contributing to the mounting evidence for the real-life effectiveness of prophylactic HPV vaccines from several countries with successfully implemented HPV vaccination programs. In addition, we present the most important safety data from large population-based cohorts evaluating potential adverse events, briefly describe the most notable HPV vaccine-related crises, and provide insight into various responses by healthcare authorities that have resulted in markedly different outcomes in the vaccination perception of the general population and, consequently, HPV vaccine uptake. Finally, global disparities in access to HPV vaccines reveal substantial gender and socioeconomic inequity that must be overcome to achieve a large population impact of HPV vaccines worldwide.
\end{abstract}

Keywords: human papillomaviruses, HPV, prophylactic HPV vaccines, recent advances

Received: 10 October 2018 | Returned for modification: 25 October 2018 | Accepted: 20 November 2018

\section{Introduction}

Prophylactic human papillomavirus (HPV) vaccination is an immensely powerful tool for preventing HPV-associated cancers, especially cervical cancer. In combination with an adequate screening program, HPV vaccination offers an opportunity to dramatically reduce cervical cancer morbidity and mortality, and to eradicate the disease in the long term. Indeed, after 12 years of implementation of HPV vaccination, some countries with consistently high coverage of the target population are already on track toward eliminating cervical cancer. For example, a recently published modeling study from Australia predicts that cervical cancer mortality will decrease to less than an age-standardized annual rate of one death per 100,000 women by 2034 (range 2025-2047) due to the exceptional effectiveness of the Australian preventive program of vaccination and screening (1).

Despite being undeniably effective, prophylactic HPV vaccines face two major obstacles nowadays: successful implementation, and sufficient and sustainable vaccine uptake. Improving access to HPV vaccines leads to a far greater increase in vaccine uptake than any other approach. Interventions such as school-based vaccination programs have been shown to achieve the highest vaccination rates while reaching the greatest number of participants (2).

In Slovenia, HPV vaccination was implemented into the national immunization program in the 2009/2010 school year for girls 11 to 12 years old (the sixth grade of primary school). In addition to the primary targeted population, the HPV vaccine is offered free of charge to the eligible catch-up cohort of all girls and young women that attended sixth grade in the 2009/2010 school year or later and have not yet been vaccinated until the end of their education. The relatively low acceptance of the HPV vaccine in Slovenia is concerning because only half of the eligible population has been vaccinated in the last 10 years and half of adolescent girls in Slovenia are missing an opportunity to prevent HPV-related cancer and precancerous lesions later in life. Such pat position is also preventing the straightforward modification of cervical cancer screening strategy for young women in Slovenia.

Every year since 2009, before the start of the HPV national immunization vaccination campaign, the Section for School, University, and Adolescent Health of the Slovenian Medical Society has been holding a professional 1-day "refresher course" with education and promotion for all healthcare workers on the frontline of HPV vaccination in Slovenia and other countries of the former Yugoslavia. This review summarizes in our opinion key peer-reviewed literature published between September 2017 and September 2018; that is, during the 2017/2018 school year in Slovenia. Most of the publications covered in this literature review were presented and discussed at the professional meeting held on September 1oth, 2018 in Ljubljana, Slovenia with the aim of refreshing and improving the knowledge of pediatricians, school medicine specialists, gynecologists, epidemiologists, and other healthcare workers responsible for HPV vaccination.

For the professional meeting and this literature review, PubMed was searched for the terms "human papillomavirus vaccine" and "HPV vaccine" with a custom date range filter of September 1st, 2017 to September 9th, 2018. During manual screening of the 955 matches retrieved in our search, we mainly focused on publications on the progress and development of novel prophylactic HPV vaccines, efficacy clinical trials evaluating various dosing schemes 
and HPV vaccination of alternative populations, studies contributing to the mounting evidence of real-life effectiveness of prophylactic HPV vaccines from several countries with successfully implemented HPV vaccination programs, publications reporting important safety data from large population-based cohorts evaluating potential adverse events, publications dealing with the most notable HPV vaccine-related crises, and those providing insight into various responses by healthcare authorities that resulted in markedly different outcomes on the vaccination perception of the general population and, consequently, HPV vaccine uptake.

\section{Candidates for novel prophylactic HPV vaccines}

At present, three prophylactic HPV vaccines are licensed: a bivalent HPV16/18 (2vHPV) vaccine, Cervarix (GlaxoSmithKline Biologicals, Belgium); a quadrivalent HPV6/11/16/18 (4vHPV) vaccine, Gardasil/Silgard (Merck \& Co., USA / Sanofi Pasteur MSD, France); and a nonavalent HPV6/11/16/18/31/33/45/52/58 (9vHPV) vaccine, Gardasily (Merck \& Co.). A novel trivalent HPV16/18/58 vaccine produced in an Escherichia coli expression system with presumably lower manufacturing cost and faster production cycle has been studied for immunogenicity and dosage effect in a rhesus macaque model, showing non-inferiority regarding strength and durability of immune responses compared to the 4VHPV vaccine (3). Such trivalent vaccine is tailored for the Asia-Pacific region because HPV58, otherwise rarely found elsewhere worldwide, ranks third among HPV types associated with cervical cancer cases reported in China, Japan, and South Korea (3).

To address the significant burden of anogenital warts worldwide, a novel HPV6/11 vaccine candidate for prevention of anogenital warts was developed. Hansenula polymorpha-derived HPV6/11 L1 proteins were correctly expressed and assembled into HPV6/11 virus-like particles (VLPs). Promising results were achieved because the vaccine was able to induce high titers of HPV6/11-specific neutralizing antibodies in mice and cynomolgus monkeys (4).

\section{Efficacy results from clinical trials}

Primary analysis of the pivotal phase 3 efficacy clinical trial of the 9vHPV vaccine in women 16 to 26 years old showed efficacy and comparable immunogenicity of the 9vHPV vaccine compared to the $4 \mathrm{vHPV}$ vaccine (5). The final analysis of this study (after 6 years of follow-up), showed $97.4 \%$ efficacy of the 9vHPV vaccine for prevention of high-grade cervical, vulvar, and vaginal disease caused by HPV31/33/45/52/58. Moreover, geometric mean titers (GMTs) of anti-HPV6/11/16/18 antibodies in the 9vHPV vaccine group were non-inferior compared to the group that received the $4 \mathrm{VHPV}$ vaccine from month 1 to 3 years following vaccination (6).

\section{Dosing schedules}

Current World Health Organization (WHO) guidelines recommend a two-dose schedule for persons starting HPV vaccination before their 15th birthday, whereas those that start vaccination on or after their 15th birthday and those with certain immunocompromising conditions should receive three doses of the vaccine $(7,8)$. The recommendation applies for all three prophylactic vaccines: 2vHPV, 4vHPV, and 9vHPV vaccine.

Early findings from a multi-center cluster randomized trial of two versus three doses of the $4 \mathrm{vHPV}$ vaccine in India indicate that even a single dose of the $4 \mathrm{vHPV}$ vaccine is immunogenic and provides at least a 7-year protection against incident HPV16/18 infections that is comparable to the two- and three-dose schedules (9). Similarly, data from the Costa Rica Vaccine Trial (CVT) on 7-year protection and immunogenicity of the $2 \mathrm{vHPV}$ vaccine further support the hypothesis that one dose of the HPV vaccine might be sufficient, which could potentially facilitate worldwide HPV vaccine implementation and enable sustainable vaccination coverage rates (10). In addition, a retrospective database study on the incidence of anogenital warts among 440,532 women and 133,394 men from the United States starting 3 months after the last dose of the $4 \mathrm{VHPV}$ vaccine showed comparable and significant effectiveness of fewer than three doses in 15- to 19-year-olds, irrespective of sex (11). However, despite promising results, further data on longterm protection beyond the first 7 years following HPV vaccination should be obtained before any policy guidelines change is considered. To address this question further, the first randomized four-arm non-inferiority trial to test the efficacy of one dose versus two doses of the 2vHPV and 9vHPV vaccines started recently in Costa Rica (clinicaltrials.gov identifier NCT03180034). The trial is sponsored by the National Cancer Institute and the Bill and Melinda Gates Foundation.

\section{Extending HPV vaccination to other populations}

The burden of cervical cancer is high in Latin America; however, HPV vaccine acceptance is suboptimal in the majority of countries, especially because adolescents are a population particularly difficult to reach in some parts of the world (12). Hence, a phase 3 controlled multicenter study was conducted in Colombia, Mexico, and Panama to evaluate the feasibility of vaccinating girls under 9 years old for the first time. A two-dose vaccination with the $2 \mathrm{vHPV}$ vaccine in girls 4 to 6 years old proved to be safe, with injection site pain being the most frequently reported solicited local symptom in the vaccinated cohort. Moreover, seroconversion for both HPV16 and HPV18 was achieved in all girls vaccinated, suggesting that a two-dose schedule with the $2 \mathrm{vHPV}$ vaccine at a pediatric age could pose a reasonable alternative to the current vaccination of adolescents (12).

In addition to the recommended vaccination for girls 11 to 12 years old, several countries offer a possibility for catch-up vaccination for older girls and women. A nested case-control study of young women from the United States showed that catch-up HPV vaccination provides the strongest protection against cervical intraepithelial neoplasia grade 2 or worse ( $\mathrm{CIN}_{2}+$ ) and $\mathrm{CIN}_{3}+$ among women that had received three doses of the $4 \mathrm{vHPV}$ vaccine and were 14 to 17 years old (relative risk [RR] 0.52, 95\% confidence interval [CI] $0.36-0.74$ and RR 0.27, 95\% CI 0.13-0.56, respectively) or 18 to 20 years old (RR 0.65, 95\% CI 0.49-0.88 and RR 0.59, 95\% CI $0.36-0.97$, respectively) at the time of the application of the first dose, whereas the vaccine did not confer protection in women $\geq$ 21 years old (RR 0.94, 95\% CI o.81-1.09) (13). Hence, these results did not support the general catch-up vaccination of women older than 21 in the United States, contradicting recent calls to extend HPV vaccination to older women. The results by Silverberg et al. (13) differed from the Australian data, showing a distinct impact of age-extended HPV vaccination, in which vaccination of multiple cohorts of women (between 12 and 26 years) generated markedly faster direct and herd effects on the incidence of anogenital warts, adding benefits that are expected to last for 20 to 70 years (14).

Regarding HPV vaccination of persons living with human im- 
munodeficiency virus (HIV) / AIDS, results from a phase 3, double-blinded, randomized controlled trial of the $4 \mathrm{vHPV}$ vaccine performed on 575 HIV-positive individuals 27 years or older do not support general HPV vaccination in this group; the $4 \mathrm{vHPV}$ vaccine was shown to have no effect on improvement of anal highgrade squamous intraepithelial lesion (HSIL) outcomes and was only $22 \%$ and $32 \%$ effective against persistent anal HPV infection or 6-month persistent oral HPV infection, respectively, while exhibiting $88 \%$ protection against persistent oral HPV infections (15). The current European AIDS Clinical Society (EACS) Guidelines thus recommend HPV vaccination with three doses of the 9vHPV vaccine for all HIV-positive individuals up to 26 years only and up to 40 years for HIV-negative men who have sex with men (MSM). However, one must be aware that vaccine efficacy (VE) is limited if HPV infection is already established at the time of first vaccine dose (16).

\section{Lack of evidence for HPV type replacement following HPV vaccination}

Concern has been raised whether the reduced prevalence of vaccine HPV types at the population level following HPV vaccination could result in clearing an ecological niche for non-vaccine HPV types. Similar to most previously published studies that did not observe an increasing prevalence of non-vaccine types, a recent large population-based, community-randomized trial was unable to identify definite type replacement patterns following vaccination with the 2vHPV vaccine (17). Moreover, an analysis of a total of 1,180 sexually active 13 - to 26 -year old women recruited from three cross-sectional studies conducted between 2006 and 2014 found no evidence of non-vaccine HPV type replacement among vaccinated women (adjusted odds ratio [AOR], 1.02; 95\% CI, 0.73-1.42) (18). In addition, data from two observational studies in Scotland and Norway also argue against increasing rates of infections with non-vaccine HPV types in their populations (19, 20). However, as shown in a recent study from the United States, the observed increase in the prevalence of non-vaccine types in unvaccinated women in some studies could be explained by demographic and behavioral differences between vaccinated and unvaccinated women rather than HPV type replacement following HPV vaccination (21).

\section{Real-life effectiveness of HPV vaccines}

The high efficacy of prophylactic HPV vaccines extends beyond comprehensive pre-licensure evaluations and large-scale clinical trials. This section presents additional findings for the mounting evidence of their real-life effectiveness that was manifested unexpectedly soon after the implementation of HPV vaccination in national immunization programs-not only in countries with very high vaccination coverage, but also in those with a lower level of HPV vaccine uptake. Because the impact of HPV vaccination at a population level mirrors the natural history of HPV infection and development of HPV-related disease, a decline in the incidence and prevalence of HPV vaccine types was the first real-life evidence of HPV vaccine effectiveness. This was soon followed by reports of a decline in the incidence of anogenital warts, which usually develop within a few months after incident infection with low-risk types HPV6 and HPV11, and precancerous lesions, which develop later. Surprisingly, in 2018, just 12 years after licensure of the first prophylactic HPV vaccine, we are already witnessing the first reports suggesting that HPV vaccination also effectively prevents HPV-related cancers and juvenile-onset recurrent respiratory papillomatosis.

\section{Decreasing incidence and cross-sectional prevalence of HPV infections}

A cross-sectional study of 2,627 young adults from the United States showed significantly reduced prevalence of oral HPV6/11/16/18 infections in the vaccinated versus unvaccinated cohort $(0.11 \%$ versus $1.61 \%$; model-adjusted $p=0.008$ ), which corresponds to an estimated $88.2 \%$ (95\% CI, 5.7-98.5\%) reduction in prevalence after adjusting for age, sex, and race (22). Because vaccine uptake is relatively low in the United States, the overall population-level effect on the prevalence of vaccine type oral HPV infection was modest $(17.0 \%)$ and was especially low in men (6.9\%), who carry the greatest burden of HPV-related oropharyngeal cancer (22).

The tremendous impact of the Australian HPV vaccination program, which initially primarily targeted girls 12 to 13 years old (commenced in 2007 with a catch-up vaccination of 14- to 26-yearold girls and young women through 2009) was shown in a recent study reporting the surveillance data with longest follow-up to date (23). In this cross-sectional study, the prevalence of HPV infections 9 years following implementation of the 4VHPV vaccine dropped from $22.7 \%$ in $2005-2007$ to only $1.5 \%$ in 2015 in women 18 to 24 years old, and from $11.8 \%$ in $2010-2012$ to $1.1 \%$ in 2015 in women 25 to 35 years old, corresponding to a $92 \%$ decrease in the prevalence of $4 \mathrm{VHPV}$ vaccine types among Australian women 18 to 35 years old (23). A similarly significant drop in the prevalence of $4 \mathrm{vHPV}$ vaccine types during the first 8 years of $\mathrm{HPV}$ vaccination from $11.5 \%$ (95\% CI, 9.1-14.4\%) in $2003-2006$ to $3.3 \%$ (95\% CI, 1.9$5.8 \%$ ) in $2011-2014$ (corresponding to a $71 \%$ decrease in the prevalence) and from $18.5 \%$ (95\% CI, $14.9-22.8 \%$ ) in $2003-2006$ to $7.2 \%$ (95\% CI, 4.7-11.1\%) in 2011-2014 was also observed among 14- to 19-year-olds and 20- to 24-year-olds from the United States, respectively (24). Both studies also observed a decrease in the prevalence of $4 \mathrm{vHPV}$ vaccine types among unvaccinated females, further confirming the strong herd immunity of HPV vaccines (23, 24).

In addition, a recent study among 2,197 Japanese women tested during cervical cancer screening between 2014 and 2016 showed high vaccine-type effectiveness of the $2 \mathrm{VHPV}$ vaccine (pooled VE against HPV16 and HPV18 of 93.3\%) and significant cross-protection against HPV31/45/52 (pooled VE of 67.7\%) (25). This study emphasizes the extent of the missed opportunity to prevent HPVrelated diseases that Japan will face due to the withdrawal of the proactive government recommendation for HPV vaccination.

Similar results are also available from various European countries. A large population-based, cross-sectional study of two non-vaccine-eligible cohorts and one vaccine-eligible cohort of Norwegian girls showed that implementation of HPV vaccination into the national program led to a $42 \%$ (95\% CI, 37-47\%) overall reduction of any HPV type and an $81 \%$ (95\% CI, 76-85\%) reduction in $4 \mathrm{VHPV}$ vaccine types in the vaccinated cohort (19). Moreover, a 7-year cross-sectional study on 8,584 samples from Scottish women showed staggering HPV16/18 prevalence reduction from $30.0 \%$ (95\% CI $26.9-33.1 \%)$ in the 1988 birth cohort to $4.5 \%(95 \%$ CI 3.5-5.7\%) in the 1995 birth cohort, corresponding to a vaccine effectiveness of $89.1 \%(85.1-92.3 \%)$ for women that were vaccinated at age 12 to 13 (20). Both the Scottish and Norwegian data showed clear evidence of cross-protection against non-vaccine HPV types as well as substantial herd protection (19, 20). In Eng- 
land, 8 years after the introduction of the $2 \mathrm{VHPV}$ vaccine in the national HPV vaccination program, the prevalence of HPV16/18 between $2010 / 2011$ and 2016 decreased from $8.2 \%$ to $1.6 \%$ in 16 - to 18 -year-olds and from $14.0 \%$ to $1.6 \%$ in 19 - to 21 -year-olds; high vaccine effectiveness for HPV16/18 (82.0\%; 95\% CI, 60.6-91.8\%) as well as for non-vaccine types HPV31/33/45 (48.7\%; 95\% CI, 20.8-66.8\%) was observed (26). Two studies evaluated the effectiveness of the $2 \mathrm{vHPV}$ vaccine in the Netherlands $(27,28)$. The first study-performed on 1,635 women, of whom 54\% were fully vaccinated-showed vaccine effectiveness against HPV16/18 and HPV31/33/45-persistent infections to be $97.7 \%$ (95\% CI, 83.5$99.7 \%$ ) and $61.8 \%$ (95\% CI, $16.7-82.5 \%$ ), respectively, without any evidence of waning protection for up to 6 years following vaccination (27). Similar findings were also obtained in the second Dutch study, on 1,087 high-risk women that attended sexually transmitted infection clinics: the adjusted vaccine effectiveness was $92.3 \%$ (95\% CI, 82.5-96.6\%) for HPV16 and 85.5\% (95\% CI 66.0-93.8\%) for HPV18, with additional protection against non-vaccine types HPV31/33/45/52/58 (vaccine effectiveness against individual HPV types ranged between $25.7 \%$ and $91.0 \%$ ) (28).

Whereas the 2vHPV vaccine was shown to be highly effective in women, the efficacy data in males for this vaccine are less abundant. Nevertheless, 4 years since the beginning of HPV vaccination of boys with the 2vHPV vaccine in Finland, the prevalence rates of HPV16/18 and HPV11/16/18/31/33/45 markedly dropped (from $2.1 \%$ to $0 \% ; p=0.02$ and from 5.3 to $0.8 \% ; p=0.002$, respectively), suggesting that the $2 \mathrm{vHPV}$ vaccine also has a protective effect in men (29).

\section{Decreasing incidence of anogenital warts}

Owing to the early implementation of catch-up vaccination in combination with stable and high vaccination coverage rates and the consequent creation of at least 25 generations of protected females, Australia was the first country to demonstrate a rapid decline in the incidence of anogenital warts in both sexes (14). The early impact of successful implementation of school-based HPV vaccination (immunization coverage rates between $78 \%$ and $81 \%$ ) in Canada was demonstrated in a study on the Quebec general population (30). The HPV vaccination program was associated with a marked reduction of anogenital warts mainly among females < 25 years old ( $45 \%$ and $19 \%$ among females 15 to 19 and 20 to 24 years old, respectively). A significant but less pronounced (21\%) reduction among males 15 to 19 years old was also observed, which could be due to herd protection and/or privately paid vaccination. HPV vaccination was also associated with an important observation: anogenital warts are being diagnosed at a later age, with the median age at diagnosis in women increasing from 27 years in 2004 to 31 years in 2012 (30).

It seems that high HPV vaccination coverage is not always mandatory for effect demonstration, as shown in a recent study from Sweden, where similar reductions in anogenital warts were observed in women (average annual percent change [AACP] range: $-8.5 \%$ to $-18.5 \%$ ) and men (AACP range: $-7.0 \%$ to $-16.6 \%$ ), in spite of relatively low HPV vaccination coverage in females (estimated at 30\%) with virtually no vaccine acceptance in males during the first years (2007-2011) of opportunistic HPV vaccination; the reductions were greatest in the youngest cohorts (31). Whereas a measurable decrease in the incidence was already noted in women in 2008-2009, the effect was postponed in men and only became apparent in 2010-2012 (31).

\section{Decreasing incidence and cross-sectional prevalence of HPV-} related precancerous lesions

According to the Victorian Cervical Cytology Registry Statistical 2015 Report, published in December 2017, the number of incident HSIL cases in screened women is continuing to decline in generations that were vaccinated against HPV, with the most profound decline observed in women $<20$ to 29 years old (32). Moreover, the final data from a 10-year follow-up study on 1,661 boys and girls that were not sexually active at the time of HPV vaccination showed that the 4vHPV vaccine is highly immunogenic, well tolerated, and highly effective with no cases of HPV6/11/16/18-related disease recorded among vaccinated individuals. These data further support gender-neutral vaccination of preadolescents and adolescents at the earliest opportunity to allow realization of the full potential of HPV vaccines (33). A 12-year long-term effectiveness follow-up of the 2,084 women from four Nordic countries vaccinated with the $4 \mathrm{VHPV}$ vaccine similarly showed no breakthrough cases of HPV16/18-related $\mathrm{CIN}_{2}+$, corresponding to $4 \mathrm{VHPV}$ vaccine effectiveness of more than $90 \%$ for at least 10 years after vaccinating women 16 to 23 years old (34).

The effectiveness of the $2 \mathrm{vHPV} / 4 \mathrm{vHPV}$ vaccine against HSIL+ in a large cohort of 22,743 Japanese women 22 to 29 years old showed a $69 \%$ lower risk for HSIL+ in vaccinated women compared to the unvaccinated cohort (35).

A Finnish Cancer Registry-based follow-up study with results on 98,561 person-years provided another important insight into the long-term protection of the 2vHPV vaccine against $\mathrm{CIN}_{3}+(36)$. The vaccine effectiveness against $\mathrm{CIN}_{3}+$ irrespective of HPV type was $66 \%$ (95\% CI 8-88\%), with four cases of CIN3 and no case of invasive cervical cancer identified in the vaccinated cohort, and 75 cases of $\mathrm{CIN}_{3}$ and four cases of invasive cervical cancer in the unvaccinated cohort during 4.5 to 10 years post enrollment. In three out of four vaccinated women with $\mathrm{CIN}_{3}+$, analysis of archival formalin-fixed, paraffin-embedded tissue samples confirmed baseline (pre-vaccination) positivity for HPV16, suggesting limited VE in subjects with preexisting HPV16 infection (36).

\section{Decreasing incidence of HPV-related cancers}

One of the last uncertainties-and the most important one-connected with HPV vaccine efficacy/effectiveness was recently revealed: HPV vaccines also effectively prevent HPV-related cancers. Based on Finnish cancer registry follow-up data for vaccinated and unvaccinated female cohorts, 10 invasive HPV-related cancer cases (eight cervical cancers, one vulvar, and one oropharyngeal) were identified in the unvaccinated women, whereas no HPV-related cancer cases were detected in vaccinated women; the incidence of other (non-HPV-related) cancers was similar among the two groups studied (37).

\section{Decreasing incidence of juvenile-onset recurrent respiratory papillomatosis}

Recurrent respiratory papillomatosis is a rare but devastating disease caused by infection with HPV6 and HPV11. It could theoretically be prevented with HPV vaccination, but this disease is not officially listed among indications for 4vHPV or 9vHPV vaccination. This might soon change because the registered national annual incidence of juvenile-onset recurrent respiratory papillomatosis declined from 0.16 per 100,000 in 2012 to 0.02 per 100,000 in 
2016 ( $p=0.034$ ) following 4VHPV vaccination program implementation in Australia (38). Of the 15 incident cases of the juvenileonset recurrent respiratory papillomatosis recorded in Australia from 2012 to 2016, none of the mothers were vaccinated against HPV (38).

\section{HPV vaccine safety: unproven rumors versus scientific evidence}

Despite a wealth of reassuring vaccine safety data, in the past few years rumors about alleged associations of HPV vaccines with poorly defined clinical syndromes with heterogeneous and often unclear etiology have been raised. Although lacking any scientific evidence, fear from such "adverse effects" and mistrust in the vaccines' safety have seriously undermined some well-established and previously very successful national HPV vaccination programs, with the most profound examples being Japan, Columbia, Denmark, and Ireland. However, the safety of HPV vaccines has been thoroughly investigated numerous times: from rigorous pre-licensing evaluations to extensive post-licensing monitoring of potential adverse reactions through active and passive surveillance in real-world settings, with no associations with any serious adverse event confirmed. Indeed, the HPV vaccine is the only vaccine alongside hepatitis $B$-virus (HBV) vaccine for which the WHO uses the term "extremely safe" to describe the level of vaccine safety (39). The following section briefly summarizes the responses related to some of the notable vaccination crises and summarizes important recent literature investigating in-depth controversial syndromes allegedly linked to the HPV vaccine.

\section{Responses to the vaccination crises}

In Japan, a small number of cases of so-called chronic regional pain syndrome (CRPS) were reported in 2013, which were subsequently proven not to be related to HPV vaccination. However, the hysteria facilitated by sensational media reporting led to almost immediate withdrawal of proactive government recommendation for HPV vaccination. Consequently, the vaccination coverage plummeted from more than $70 \%$ (recorded in 5 consecutive years) to less than $1 \%(35,40)$. To investigate any potential association between the HPV vaccine and reported post-vaccination symptoms, the city of Nagoya conducted a large-scale questionnairebased survey among female residents born between 1994 and 2001 (41). In total, almost 30,000 residents responded; the results showed no significant increase in occurrence of any of the 24 reported post-HPV vaccination symptoms (41). Regrettably, 5 years after suspension, the government recommendation still has not been reinstated, one recent reason given being the lack of reliable vaccine effectiveness data in a Japanese population (25). However, vaccine effectiveness was clearly confirmed in studies by Kudo et al. (25) and Konno et al. (35), described in detail above, further emphasizing the enormous missed opportunity to prevent HPV-related disease in Japanese women and men. Interestingly, a study by Yamaguchi et al. found only moderate validity of self-reported information regarding HPV vaccination status; combined with a low negative predictive value ( $54.7 \%$ ), this may even lead to underestimation of the effectiveness and safety of the HPV vaccine (42). Thus, more reliable surveillance methods, such as a national HPV vaccine registry, should be established by the government for assessing HPV vaccination status in Japan (42). In the past, politicized transitions by the Japanese government in vaccination policy regarding measles, mumps, and rubella (MMR) vaccination caused important herd immunity gaps, resulting in outbreaks with 17,050 cases of rubella overall and 45 cases of congenital rubella syndrome reported between 2012 and 2014 (43). Unfortunately, in Japan history is repeating itself because the responsible authorities are failing to learn from the mistakes of the past and are refusing to support HPV vaccination despite the strong evidence of the vaccine's effectiveness and safety (43).

Denmark used to have one of the most successful national HPV vaccination programs in the world, with vaccination coverage reaching 80 to $90 \%$ of the target population for several years in a row. However, in 2012, a number of cases of postural orthostatic tachycardia syndrome (POTS) and similar syndromes in young women were reported and hypothesized to be associated with HPV vaccination; possible adverse effects were highlighted in the television documentary De vaccinerede piger (The Vaccinated Girls), and a negative attitude toward HPV vaccination was widely disseminated through social media (44). The HPV vaccination coverage decreased dramatically to only 20 to $30 \%$. Following a review from the European Medicines Agency (EMA), which found no evidence for a causal association between HPV vaccination and POTS or related syndromes, and after a concerted government effort called "StopHPV" was initiated to disseminate scientific evidence regarding HPV vaccination, in June 2018 the vaccination coverage was back to $73 \%$. In addition, boys in Denmark are now offered free vaccination as well (44). However, a few generations of girls were vaccinated at age 15 to 16 instead of at age 12, when more than a third of them were already sexually active; the initial prospect of attaining herd immunity was thus temporarily postponed by approximately 4 to 5 years in Denmark (44).

The vaccination crisis in Ireland was handled with rapid response and intensive activities. The uptake of the first dose of the vaccine decreased to $50 \%$ in 2016-2017 from the initial 80 to $90 \%$ in 2010-2015 due to the strong social media campaign of anti-vaccine lobby groups, with the highlight in the form of a documentary broadcast on national television (45). To regain parental trust, the Irish National Immunization Office immediately established a steering group of concerned organizations, intensified activity on social media, revised print and online materials, and implemented a comprehensive training program for health professionals, including e-learning modules (45). In addition, in 2017 the HPV Vaccination Alliance was launched, consisting of over 35 different organizations working in health, women's rights, child welfare, and wider civil society, committed to raising awareness of HPV vaccination. Interestingly, Irish Minister of Health Simon Harris was the driving force behind many concerted Irish efforts. Forming powerful cross-sectoral alliances reversed the decreasing trend and led to rapid improvement in vaccine uptake to $55.8 \%$ in 2016/17 and 61.7\% in 2017/18 (45).

\section{Post-licensing real-life safety data on HPV vaccines}

In addition to the EMA's safety review in 2015, the US Centers for Disease Control and Prevention (CDC) and US Food and Drug Administration (FDA) evaluated the reports of POTS following any type of HPV vaccination in the Vaccine Adverse Event Reporting System (VAERS) database from June 2006 to August 2015 (46). Among 40,735 VAERS reports following HPV vaccination, 29 POTS reports were identified that fully met diagnostic criteria; of these, $20(68.9 \%)$ reports documented a history of preexisting medical conditions. Approximately one POTS case is reported for every 6.5 
million HPV vaccine doses distributed in the United States, with no empirical Bayesian data mining safety signals for POTS and HPV vaccination detected (46).

A retrospective cohort study of 199,078 female patients 11 to 34 years old describing primary ovarian insufficiency (POI) incidence and estimating risk after adolescent vaccination found 46 confirmed idiopathic POI cases in the Kaiser Permanente Northwest electronic health records (47). Only one confirmed case received the HPV vaccine 23 months before the first clinical evaluation of POI, among a population of 58,871 young women that received the HPV vaccine during the study period (47). These findings confirm there is no statistically significant elevated risk of POI after HPV vaccination.

A population-based retrospective cohort study using Ontario’s administrative health and vaccination databases from 2007 to 2013, which included 290,939 girls 12 to 17 years old eligible for vaccination, showed no increased risk for developing 12 defined autoimmune diseases (including Bell's palsy, optic neuritis, and Graves' disease) following 4vHPV vaccination (48).

A national cohort study of 568,410 boys born in Denmark between 1988 and 2006, followed for four million person-years from 2006 to 2016, used nationwide registers to obtain individual-level information about received doses of the $4 \mathrm{VHPV}$ vaccine and hospital records for 39 different autoimmune diseases, 12 neurological diseases, and venous thromboembolism (49). Among 7,384 boys that received at least one dose of the $4 \mathrm{VHPV}$ vaccine at age 10 to 17 , none of the 52 individual outcomes studied appeared to occur in excess, providing population-based reassurance that the HPV vaccine is unlikely to be associated with autoimmune and neurological diseases or venous thromboembolism in boys (49).

The association between HPV vaccination and risk of chronic fatigue syndrome / myalgic encephalomyelitis (CFS/ME) was investigated in a nationwide register-based study in Norway; in addition, medical history in relation to both risk of CFS/ME and HPV vaccine uptake was assessed (50). A similar annual increase in the incidence rate of CFS/ME between 2009 and 2014 was observed among 824,133 boys and girls 10 to 17 years old; HPV vaccination was not associated with CFS/ME, but the risk of CFS/ME increased with increasing number of previous hospital contacts (50).

The occurrence of 38 preselected outcomes in almost 135,000 vaccinated girls 11 to 15 years old in Finland between 2013 and 2016 was investigated in a nationwide register-based cohort study (51). Again, no significantly increased risk after $2 \mathrm{vHPV}$ vaccination was found for the defined outcomes, including controversial POTS and CRPS, whereas chronic fatigue syndrome / systemic exertion intolerance disease (CFS/SEID) showed an increase, but a similar increase was also observed in unvaccinated males (51).

Autoimmune/autoinflammatory syndrome induced by adjuvants (ASIA) was first described in 2011 and defined as a group of disorders caused by aluminum-containing vaccine adjuvants, infections, and exposure to chemicals such as silicone or squalene; however, a recently published review of its diagnostic criteria actually refuted the existence of this syndrome (52). The review examined relevant human data, particularly from patients undergoing allergen-specific immunotherapy, receiving 100 to 500 times higher concentrations of injected aluminum over 3 to 5 years, compared with HBV vaccine and HPV vaccine recipients; however, patients undergoing allergen-specific immunotherapy were shown to have a lower incidence of autoimmune diseases. Therefore, current scientifically valid data do not support either the sole existence of ASIA syndrome or its etiological link with vaccine adjuvants containing aluminum (52).

An excellent safety profile of HPV vaccines was further confirmed in a Cochrane Library systematic review of 26 randomized clinical trials, showing with high certainty that the risk of serious adverse events in HPV-vaccinated groups was comparable to those receiving placebo or any other vaccine (53). The number of deaths was low overall, with a higher number of deaths in older vaccinated women, but no pattern in the cause or timing of death has been established and the deaths have been judged not to be related to the HPV vaccine. No increased risk of miscarriage or pregnancy termination was observed (53).

\section{Implementation challenges}

Despite the exceptional potential for preventing HPV-related diseases, especially cervical cancer, and despite being one of the most studied vaccines in history, significant global disparities in access to the HPV vaccine limit the impact that could have been achieved with optimal vaccination coverage.

As of October 4th, 2018, the HPV vaccine has been introduced in the national immunization program (including full or partial introduction) in 91 countries, which still comprises less than half the countries in the world (54). Unfortunately, the vaccination coverage varies substantially and remains low in many settings, the main obstacles identified being i) high vaccine cost; ii) insufficient healthcare infrastructure in developing countries, often with a well-established system of infant and child vaccination, but no existing platforms for adolescent vaccination; iii) reaching the target population and maintaining high vaccination coverage over years; and iv) vaccine hesitancy and alleged safety concerns, often driven by controversial stories spread through social media, which are not handled appropriately by health authorities.

Although the majority of global cervical cancer burden occurs in low- and lower-middle-income countries, girls and women from these countries are the least likely to be vaccinated. By June 2016, $71 \%$ of high-income countries, $35 \%$ of upper-middle-income countries, $8 \%$ of lower-middle-income countries, and $6 \%$ of lowincome countries had introduced the HPV vaccine into their national immunization schedule (55). With concerted support of global partners such as the WHO, Pan American Health Organization (PAHO), and Gavi, the Vaccine Alliance, the availability of HPV vaccines is increasing due to subsidized prices in recent years, and the number of low- and lower-middle-income countries with implemented HPV vaccination programs is also increasing.

A recent review of HPV vaccination experience in demonstration projects or national programs in 45 low- and middle-income countries between 2007 and 2016 has shown that in these countries final dose coverage estimates were all well above 50\% (most between $70 \%$ and 90\%), and showed no trend over time (56). The majority of delivery strategies included schools as a vaccination venue, and, in countries with school enrolment rates below $90 \%$, inclusion of strategies to reach out-of-school girls contributed to obtaining high coverage compared to school-based-only strategies (56). Ironically, despite immeasurably inferior healthcare infrastructure, the HPV vaccination coverage achieved in several low- and middle-income countries is much higher than in several high-income countries, such as France, Germany, Sweden, and the United States.

One successful example of HPV vaccine implementation is South Africa. In a country where one in 26 women develop cervical cancer in their lifetime, during implementation of a national 
school-based HPV vaccination program in 2014 over 350,000 girls $>9$ years old attending the fourth grade were vaccinated in more than 16,000 public schools, which represented $86.6 \%$ vaccination coverage of age-eligible students (57). The campaign's successes were attributed to careful planning and coordination, and strong leadership from the National Department of Health (57).

In addition to wealth inequity regarding access to HPV vaccination, gender inequity further hampers the potential of the HPV vaccine to eliminate HPV-related diseases in both genders. HPV has a long history of being "feminized"; intersected by science, politics, economics, and gender norms (58). Initially, the HPV vaccine was approved for adolescent females based on available data and the social context, and it was later on further feminized through marketing, leaving vaccine benefits for men mainly unnoticed (58). However, both genders are responsible for HPV transmission and both genders are affected by HPV-associated diseases. In fact, although cervical cancer accounts for the majority of HPVassociated cancers globally, in 2015 oropharyngeal cancer was the most common HPV-associated cancer in the United States, with the incidence in men exceeding the incidence of cervical cancer in women (59). Gender-neutral vaccination would have a significantly greater impact on the prevention of cervical cancer, as well as prevention of other HPV-associated diseases, including genital warts, due to better and faster establishment of herd immunity.

Following FDA approval of the $4 \mathrm{VHPV}$ vaccine for use in males 9 to 26 years old in October 2009, the United States was the first large country to expand its national immunization program to include boys in routine HPV vaccination in 2011. However, the uptake of the HPV vaccine remains relatively low for both genders because in 2016 only $49.5 \%$ female and $37.5 \%$ male adolescents 13 to 17 years old were fully vaccinated, with a slight increase of vaccine uptake in 2017 , with $53.1 \%$ of female and $44.3 \%$ of male adolescents receiving full vaccination (60). By 2015, five additional countries (Canada, Australia, Israel, Trinidad and Tobago, and Hungary) expanded their recommendation for HPV vaccination to also include boys, and as of 2018 the number of countries has already risen to 27 , indicating an encouraging trend toward broader availability of the HPV vaccine for both genders (61). Australia, as the best example of successful implementation of the HPV vac- cine for both genders, managed first to achieve high coverage of female adolescents, with three-dose coverage at age 15 being consistently over $70 \%$ since the beginning of HPV vaccination and having even increased to $78.6 \%$ in 2016 (62). HPV vaccination of male adolescents started in 2013 and reached $72.9 \%$ three-dose coverage at age 15 in just 3 years; both achievements reflect ongoing evaluation and improvements to school-based vaccine delivery systems in Australia (62).

The impact of gender-neutral versus girls-only HPV vaccination was recently studied in a community-randomized clinical trial, randomizing the entire 1992-1995 birth cohorts in 33 Finnish communities into three arms: i) gender-neutral HPV vaccination, ii) HPV vaccination of girls and HBV vaccination of boys, and iii) gender-neutral HBV vaccination $(63,64)$. The results showed that the gender-neutral strategy enhanced the effectiveness of HPV vaccination for cross-protection against HPV types HPV31/33/35/45 with low to moderate coverage, and that high coverage in males was also crucial for providing a substantial public health benefit to unvaccinated females $(63,64)$.

\section{Conclusions}

To seize its full potential and achieve a larger and earlier population impact, the HPV vaccine should be introduced into national or regional immunization schemes in a gender-neutral manner and possibly including older catch-up cohorts. Moreover, coverage of the target population should be consistently high over the years. This can only be achieved with appropriate support of national/regional healthcare officials and authorities, good healthcare infrastructure, and excellent knowledge among healthcare workers, who are responsible for education, promotion, organization, and delivery of the HPV vaccine. Equally important, general public confidence in the HPV vaccine should remain positive; the impact of rumors and misinformation should be appropriately and rapidly handled by healthcare authorities, possible adverse effects should be reported accurately, and their significance and potential link to the HPV vaccine should be investigated using adequate and proven scientific methodology.

\section{References}

1. Hall MT, Simms KT, Lew JB, Smith MA, Brotherton JM, Saville M, et al. The projected timeframe until cervical cancer elimination in Australia: a modelling study. Lancet Public Health. 2018; [Epub ahead of print].

2. Walling EB, Benzoni N, Dornfeld J, Bhandari R, Sisk BA, Garbutt J, et al. Interventions to improve HPV vaccine uptake: a systematic review. Pediatrics. 2016;138. pii: e20153863.

3. Yin F, Wang Y, Chen N, Jiang D, Qiu Y, Wang Y, et al. A novel trivalent HPV 16/18/58 vaccine with anti-HPV 16 and 18 neutralizing antibody responses comparable to those induced by the Gardasil quadrivalent vaccine in rhesus macaque model. Papillomavirus Res. 2017;3:85-90.

4. Yu Y, Guo J, Li D, Liu Y, Yu Y, Wang L. Development of a human papillomavirus type $6 / 11$ vaccine candidate for the prevention of condyloma acuminatum. Vaccine. 2018;36:4927-34.

5. Joura EA, Giuliano AR, Iversen OE, Bouchard C, Mao C, Mehlsen J, et al. A 9-valent HPV vaccine against infection and intraepithelial neoplasia in women. $\mathrm{N}$ Engl J Med. 2015;372:711-23.

6. Huh WK, Joura EA, Giuliano AR, Iversen OE, de Andrade RP, Ault KA, et al. Final efficacy, immunogenicity, and safety analyses of a nine-valent human papillomavirus vaccine in women aged $16-26$ years: a randomised, double-blind trial. Lancet. 2017;390:2143-59.

7. Meites E, Kempe A, Markowitz LE. Use of a 2-dose schedule for human papillomavirus vaccination-updated recommendations of the Advisory Committee on Immunization Practices. MMWR Morb Mortal Wkly Rep. 2016;65:1405-8.
8. World Health Organization. Human papillomavirus vaccine: WHO position paper, May 2017. Weekly Epidemiol Rec. 2017;92:241-98.

9. Sankaranarayanan R, Joshi S, Muwonge R, Esmy PO, Basu P, Prabhu P, et al. Can a single dose of human papillomavirus (HPV) vaccine prevent cervical cancer? Early findings from an Indian study. Vaccine. 2018;36:4783-91.

10. Safaeian M, Sampson JN, Pan Y, Porras C, Kemp TJ, Herrero R, et al. Durability of protection afforded by fewer doses of the HPV16/18 vaccine: the CVT trial. J Natl Cancer Inst. 2018;110.

11. Zeybek B, Lin YL, Kuo YF, Rodriguez AM. The impact of varying numbers of quadrivalent human papillomavirus vaccine doses on anogenital warts in the United States: a database study. J Low Genit Tract Dis. 2018;22:189-94.

12. Lin L, Parra MM, Sierra VY, Cespedes AS, Granados MA, Luque A, et al. Safety and immunogenicity of the HPV-16/18 ASo4-adjuvanted vaccine in 4-6-year-old girls: results to month 12 from a randomized trial. Pediatr Infect Dis J. 2018;37: e93-e102.

13. Silverberg MJ, Leyden WA, Lam JO, Gregorich SE, Huchko MJ, Kulasingam S, et al. Effectiveness of catch-up human papillomavirus vaccination on incident cervical neoplasia in a US health-care setting: a population-based case-control study. Lancet Child Adolesc Health. 2018;2:707-14.

14. Drolet M, Laprise JF, Brotherton JML, Donovan B, Fairley CK, Ali H, et al. The impact of human papillomavirus catch-up vaccination in Australia: implications for introduction of multiple age cohort vaccination and postvaccination data interpretation. J Infect Dis. 2017;216:1205-9. 
15. Wilkin TJ, Chen H, Cespedes MS, Leon-Cruz JT, Godfrey C, Chiao EY, et al. A randomized, placebo-controlled trial of the quadrivalent human papillomavirus vaccine in human immunodeficiency virus-infected adults aged 27 years or older: AIDS Clinical Trials Group Protocol A5298. Clin Infect Dis. 2018;67:1339-46.

16. European AIDS Clinical Society Guidelines v9.o October 2017 [Internet]. 2018 [cited 2018 Nov 18]. Available from: www.eacsociety.org/files/guidelines_9.0english.pdf.

17. Gray P, Palmroth J, Luostarinen T, Apter D, Dubin G, Garnett G, et al. Evaluation of HPV type-replacement in unvaccinated and vaccinated adolescent femalespost-hoc analysis of a community-randomized clinical trial (II). Int J Cancer. 2018;142:2491-500.

18. Saccucci M, Franco EL, Ding L, Bernstein DI, Brown D, Kahn JA. Non-vaccine-type human papillomavirus prevalence after vaccine introduction: no evidence for type replacement but evidence for cross-protection. Sex Transm Dis. 2018;45:260-5.

19. Feiring B, Laake I, Christiansen IK, Hansen M, Stålcrantz J, Ambur OH, et al. Substantial decline in prevalence of vaccine-type and nonvaccine-type human papillomavirus (HPV) in vaccinated and unvaccinated girls 5 years after implementing HPV vaccine in Norway. J Infect Dis. 2018;218:1900-10.

20. Kavanagh K, Pollock KG, Cuschieri K, Palmer T, Cameron RL, Watt C, et al. Changes in the prevalence of human papillomavirus following a national bivalent human papillomavirus vaccination programme in Scotland: a 7-year cross-sectional study. Lancet Infect Dis. 2017;17:1293-302.

21. Ding L, Widdice LE, Kahn JA. Differences between vaccinated and unvaccinated women explain increase in non-vaccine-type human papillomavirus in unvaccinated women after vaccine introduction. Vaccine. 2017;35:7217-21.

22. Chaturvedi AK, Graubard BI, Broutian T, Pickard RKL, Tong ZY, Xiao W, et al. Effect of prophylactic human papillomavirus (HPV) vaccination on oral HPV infections among young adults in the United States. J Clin Oncol. 2018;36:262-7.

23. Machalek DA, Garland SM, Brotherton JML, Bateson D, McNamee K, Stewart M, et al. Very low prevalence of vaccine human papillomavirus types among 18- to 35-year old Australian women 9 years following implementation of vaccination. J Infect Dis. 2018;217:1590-600.

24. Oliver SE, Unger ER, Lewis R, McDaniel D, Gargano JW, Steinau M, et al. Prevalence of human papillomavirus among females after vaccine introduction-National Health and Nutrition Examination Survey, United States, 2003-2014. J Infect Dis. 2017;216:594-603.

25. Kudo R, Yamaguchi M, Sekine M, Adachi S, Ueda Y, Miyagi E, et al. Bivalent human papillomavirus vaccine effectiveness in a Japanese population: high vaccine-type-specific effectiveness and evidence of cross-protection. J Infect Dis. 2018; in press.

26. Mesher D, Panwar K, Thomas SL, Edmundson C, Choi YH, Beddows S, et al. The impact of the national HPV vaccination program in England using the bivalent HPV vaccine: surveillance of type-specific HPV in young females, 2010-2016. J Infect Dis. 2018;218:911-21.

27. Donken R, King AJ, Bogaards JA, Woestenberg PJ, Meijer CJLM, de Melker HE. High effectiveness of the bivalent human papillomavirus (HPV) vaccine against incident and persistent HPV infections up to 6 years after vaccination in young Dutch women. J Infect Dis. 2018;217:1579-89.

28. Woestenberg PJ, King AJ, van Benthem BHB, Donken R, Leussink S, van der Klis FRM, et al. Bivalent vaccine effectiveness against type-specific HPV positivity: evidence for cross-protection against oncogenic types among Dutch STI clinic visitors. J Infect Dis. 2018;217:213-22.

29. Lehtinen T, Söderlund-Strand A, Petäjä T, Eriksson T, Jokiranta S, Natunen K, et al. Human papillomavirus (HPV) prevalence in male adolescents 4 years after HPV-16/18 vaccination. J Infect Dis. 2017;216:966-8.

30. Steben M, Ouhoummane N, Rodier C, Sinyavskaya L, Brassard P. The early impact of human papillomavirus vaccination on anogenital warts in Québec, Canada. J Med Virol. 2018;90:592-8.

31. Herweijer E, Ploner A, Sparén P. Substantially reduced incidence of genital warts in women and men six years after HPV vaccine availability in Sweden. Vaccine. 2018;36:1917-20.

32. Victorian Cervical Cytology Registry. Statistical report 2015 [Internet]. [cited 2018 Oct 1]. Available from: https://www.vccr.org/site/VCCR/filesystem/ documents/dataandresearch/StatisticalReports/17030_VCS_StatsReport15_ ART.3.pdf.

33. Ferris DG, Samakoses R, Block SL, Lazcano-Ponce E, Restrepo JA, Mehlsen J, et al. 4-valent human papillomavirus (4VHPV) vaccine in preadolescents and adolescents after 10 years. Pediatrics. 2017;140.

34. Kjaer SK, Nygård M, Dillner J, Brooke Marshall J, Radley D, Li M, et al. A 12-year follow-up on the long-term effectiveness of the quadrivalent human papillomavirus vaccine in 4 Nordic countries. Clin Infect Dis. 2018;66:339-45.

35. Konno R, Konishi H, Sauvaget C, Ohashi Y, Kakizoe T. Effectiveness of HPV vaccination against high grade cervical lesions in Japan. Vaccine. 2018;36:7913-5.

36. Lehtinen M, Lagheden C, Luostarinen T, Eriksson T, Apter D, Harjula K, et al. Ten-year follow-up of human papillomavirus vaccine efficacy against the most stringent cervical neoplasia end-point-registry-based follow-up of three cohorts from randomized trials. BMJ Open. 2017;7:e015867.

37. Luostarinen T, Apter D, Dillner J, Eriksson T, Harjula K, Natunen K, et al. Vaccination protects against invasive HPV-associated cancers. Int J Cancer. 2018;142: 2186-7.
38. Novakovic D, Cheng ATL, Zurynski Y, Booy R, Walker PJ, Berkowitz R, et al. A prospective study of the incidence of juvenile-onset recurrent respiratory papillomatosis after implementation of a national HPV vaccination program. J Infect Dis. 2018;217:208-12.

39. Meeting of the Global Advisory Committee on Vaccine Safety, 7-8 June 2017. Wkly Epidemiol Rec. 2017;92:393-402.

40. Hanley SJ, Yoshioka E, Ito Y, Kishi R. HPV vaccination crisis in Japan. Lancet. 2015;385:2571.

41. Suzuki S, Hosono A. No association between HPV vaccine and reported postvaccination symptoms in Japanese young women: results of the Nagoya study. Papillomavirus Res. 2018;5:96-103.

42. Yamaguchi M, Sekine M, Kudo R, Adachi S, Ueda Y, Miyagi E, et al. Differential misclassification between self-reported status and official HPV vaccination records in Japan: implications for evaluating vaccine safety and effectiveness. Papillomavirus Res. 2018;6:6-10.

43. Tanaka Y, Ueda Y, Yoshino K, Kimura T. History repeats itself in Japan: failure to learn from rubella epidemic leads to failure to provide the HPV vaccine. Hum Vaccin Immunother. 2017;13:1859-60.

44. Lynge E, Skorstengaard M, Lübker CL, Thamsborg L. HPV-vaccination impact in Denmark: is the vaccine working? Expert Rev Vaccines. 2018;17:765-7.

45. Corcoran B, Clarke A, Barrett T. Rapid response to HPV vaccination crisis in Ireland. Lancet. 2018;391:2103.

46. Arana J, Mba-Jonas A, Jankosky C, Lewis P, Moro PL, Shimabukuro TT, et al. Reports of postural orthostatic tachycardia syndrome after human papillomavirus vaccination in the Vaccine Adverse Event Reporting System. J Adolesc Health. 2017;61:577-82.

47. Naleway AL, Mittendorf KF, Irving SA, Henninger ML, Crane B, Smith N, et al. Primary ovarian insufficiency and adolescent vaccination. Pediatrics. 2018;142: e20180943.

48. Liu EY, Smith LM, Ellis AK, Whitaker H, Law B, Kwong JC, et al. Quadrivalent human papillomavirus vaccination in girls and the risk of autoimmune disorders: the Ontario Grade 8 HPV Vaccine Cohort Study. CMAJ. 2018;190:E648-55.

49. Frisch M, Besson A, Clemmensen KKB, Valentiner-Branth P, Mølbak K, Hviid A. Quadrivalent human papillomavirus vaccination in boys and risk of autoimmune diseases, neurological diseases and venous thromboembolism. Int J Epidemiol. 2018;47:634-41.

50. Feiring B, Laake I, Bakken IJ, Greve-Isdahl M, Wyller VB, Håberg S, et al. HPV vaccination and risk of chronic fatigue syndrome / myalgic encephalomyelitis: a nationwide register-based study from Norway. Vaccine. 2017;35:4203-12.

51. Skufca J, Ollgren J, Artama M, Ruokokoski E, Nohynek H, Palmu AA. The association of adverse events with bivalent human papilloma virus vaccination: a nationwide register-based cohort study in Finland. Vaccine. 2018;36:5926-33.

52. Ameratunga R, Gillis D, Gold M, Linneberg A, Elwood JM. Evidence refuting the existence of autoimmune/autoinflammatory syndrome induced by adjuvants (ASIA). J Allergy Clin Immunol Pract. 2017;5:1551-5.e1.

53. Arbyn M, Xu L, Simoens C, Martin-Hirsch PPL. Prophylactic vaccination against human papillomaviruses to prevent cervical cancer and its precursors (review). Cochrane Database Syst Rev. 2018;5:CDoo9069.

54. World Health Organization. Vaccine in National Immunization Programme Update-World Health Organization [Internet]. 2018 [cited 2018 Nov 18]. Available from: http://www.who.int/immunization/monitoring_surveillance/VaccinelntroStatus.pptx.

55. World Health Organization. Meeting of the Strategic Advisory Group of Experts on Immunization, October 2016-conclusions and recommendations. Wkly Epidemiol Rec. 2016;91:561-82.

56. Gallagher KE, Howard N, Kabakama S, Mounier-Jack S, Burchett HED, LaMontagne DS, et al. Human papillomavirus (HPV) vaccine coverage achievements in low and middle-income countries 2007-2016. Papillomavirus Res. 2017;4:728.

57. Delany-Moretlwe S, Kelley KF, James S, Scorgie F, Subedar H, Dlamini NR, et al. Human papillomavirus vaccine introduction in South Africa: implementation lessons from an evaluation of the national school-based vaccination campaign. Glob Health Sci Pract. 2018;6:425-38.

58. Daley EM, Vamos CA, Thompson EL, Zimet GD, Rosberger Z, Merrell L, et al. The feminization of HPV: how science, politics, economics and gender norms shaped U.S. HPV vaccine implementation. Papillomavirus Res. 2017;3:142-8.

59. Van Dyne EA, Henley SJ, Saraiya M, Thomas CC, Markowitz LE, Benard VB. Trends in human papillomavirus-associated cancers-United States, 1999-2015. MMWR Morb Mortal Wkly Rep. 2018;67:918-24.

6o. Walker TY, Elam-Evans LD, Yankey D, Markowitz LE, Williams CL, Mbaeyi SA, et al. National, regional, state, and selected local area vaccination coverage among adolescents aged 13-17 years-United States, 2017. MMWR Morb Mortal Wkly Rep. 2018;67:909-17.

61. Phillips M, Morais E, Kothari S, Tantri A, Parellada Cl, Cashat M, et al. Evolution of gender-neutral HPV vaccination in national immunization programs around the world. 32nd International Papillomavirus Conference; 2018 Oct 2-6; Sydney, Australia. Poster IPVC8-0348.

62. Brotherton JML, Bloem PN. Population-based HPV vaccination programmes are safe and effective: 2017 update and the impetus for achieving better global coverage. Best Pract Res Clin Obstet Gynaecol. 2018;47:42-58. 
63. Lehtinen M, Luostarinen T, Vänskä S, Söderlund-Strand A, Eriksson T, Natunen $\mathrm{K}$, et al. Gender-neutral vaccination provides improved control of human papillomavirus types $18 / 31 / 33 / 35$ through herd immunity: results of a community randomized trial (III). Int J Cancer. 2018;143:2299-310.
64. Lehtinen M, Söderlund-Strand A, Vänskä S, Luostarinen T, Eriksson T, Natunen $\mathrm{K}$, et al. Impact of gender-neutral or girls-only vaccination against human papillomavirus-results of a community-randomized clinical trial (I). Int J Cancer. 2018;142:949-58. 